\title{
Surfactant abnormalities in ALTE and SIDS
}

\author{
I B Masters, J Vance, B A Hills
}

\begin{abstract}
Abnormalities in the relative concentrations of the components of surfactant have been implicated in prolonged expiratory apnoea (PEA) and sudden infant death syndrome (SIDS). Controversy has, however, surrounded these findings, as they may be secondary to terminal life events. In this study the physical properties of surfactant were measured in children with recurrent apparent life threatening events (ALTEs), PEA, and SIDS. Bronchial lavage samples were obtained from 21 children with recurrent ALTEs, two SIDS victims, and 26 control patients. Lipid components were immediately elutriated from these samples with liquid chloroform. The physical properties of the extracted surfactant were studied on a Langmuir trough in which the area (A) of the monolayer was cycled continuously as the surface tension $(\gamma)$ was measured by the Wilhelmy method using a platinum 'flag'. The investigators performing these tests were unaware of the clinical diagnosis. Twenty one of 23 patients displayed abnormal physical properties while seven of 26 controls displayed similar abnormalities. These abnormalities were partially inverted hysteresis (figure of eight) loops and inverted (anticlockwise) loops that also generally exhibited less hysteresis. Of the 26 controls 20 exhibited a wide hysteresis pattern that cycled in a normal (clockwise) direction. These differences were significantly different. It is concluded that children with recurrent ALTEs have definable abnormalities in the physical properties of surfactant and that these findings may provide a sensitive means of identifying those at risk of recurrent ALTEs and SIDS.

(Arch Dis Child 1994; 71: 501-505)
\end{abstract}

The cause or causes of sudden infant death syndrome (SIDS) remain a major medical and scientific dilemma. The National Institutes of Health and Human Development cooperative epidemiological study of risk factors suggests that up to $7 \%$ of SIDS victims have had prolonged apnoeic episodes or an apparent life threatening event (ALTE) before death. ${ }^{1}$ While the clear inference is that ALTEs may have a causative role in SIDS or represent part of a pathophysiological continuum with SIDS, this association is low.

Anatomical pathology studies in children dying from SIDS implicate disturbance in micromechanical processes and surfactant function as the major factor responsible for mechanical stability of the distal airspaces. ${ }^{2}$ Reduced concentrations of surfactant, in particular disaturated phosphatidylcholine (DPPC) have been described in infants with PEA and SIDS. ${ }^{3-9}$ Southall et al have implicated low concentrations of DPPC and postulated lung mechanic, neurosensory, and pulmonary vascular mechanisms for these events. 56

Previously we have shown that both an infant and young child with prolonged expiratory apnoea had significant quantitative and qualitative abnormalities in their surfactant. ${ }^{10}$ These findings were similar to the low concentrations of DPPC found in other studies. $^{2-5}$ The reliability of such findings, however, is questionable in both those and our own observations, as the ability to extract surfactant with lung washings in a standardised fashion from the living subject's lung is difficult. Despite this, there were clear cut abnormalities and, most importantly, the results were reproducible over time. This suggests that there may be a sustained abnormality and that it is not greatly influenced by intervening events, such as hypoxia. Our previous study also found greatly reduced hysteresis in the relationship between surface tension $(\gamma)$ and surface area (A), with the normal clockwise $\gamma$ :A loop actually reversing and cycling anticlockwise for the index cases. The reason for these changes is not clear although inverse hysteresis has been reported for fatigued monolayers of surfactant, ${ }^{11}$ while contact angle changes could also produce similar loops. ${ }^{12}$

These initial findings clearly supported the previous hypotheses of Morley et $a l^{3}$ and Southall et $a l^{56}$ in that abnormal surfactant function may result in altered receptorcontroller feedback regulation of functional residual capacity, thus promoting hypoxia and possibly further disorganising respirations to produce apnoea.

While long term prospective studies are being planned, we present our findings from infants presenting with recurrent or severe ALTEs to assess further the likelihood of an association of PEA (prolonged expiratory apnoea), ALTEs, and SIDS on the basis of surfactant abnormalities.

\section{Subjects and methods}

\section{INDEX CASES}

All patients were previously investigated by consultant paediatricians before referral to the respiratory unit for further assessment and investigation. The index cases comprised 23 infants and children admitted to the Mater Children's Hospital for investigation of recurrent or severe ALTEs from 1991-3. This 
group represents approximately $30 \%$ of all ALTE patients referred for respiratory assessment but would represent a much smaller percentage of infants referred to the Mater Children's Hospital for ALTEs over the same time period. All were well when investigated with a bronchoscopic procedure at an elective time at least two weeks after their admission for the ALTE. This did not include two infants who died from SIDS whose samples were obtained by deep bronchial lavage through the endotracheal tube during the resuscitation process. Four of the infants studied were siblings of SIDS victims and three of the four had had recurrent ALTEs without a definable cause. Two had a tracheostomy in situ for 'dynamic' or 'functional' (non-anatomical) upper airway obstruction. No infant had an acute infective process. There were 12 infants and children in whom it was logistically possible to undertake polysomnographic sleep studies at the time of hospital investigation for their ALTE.

DIAGNOSIS OF ALTE

The diagnosis of ALTE was defined by a consultant paediatrician in accordance with a generally accepted definition ${ }^{1}$ and consisted of a convincing history of the sudden onset of colour change, tone change, and apnoea that required vigorous stimulation and, as such, was frightening to the parent or caregiver. As most infants were referred for investigation after their initial ALTE, they usually had apnoea alarms in use. Consequently the parental history of ALTE was often augmented by those events defined by an apnoea alarm. Other conditions were defined by clinical assessments and usual investigatory techniques.

\section{CONTROL CASES}

Twenty six children acted as controls. Twenty three had undergone diagnostic bronchoscopy over the same period of time for disorders such as infantile larynx (laryngomalacia) or other structural abnormalities of the larynx or airway. Three children had a tracheostomy in situ for severe upper airway obstruction.

\section{BRONCHOSCOPIC PROCEDURE}

In no cases were the bronchoscopies performed 'just to obtain surfactant'. Indeed all patients were referred from general paediatricians to the respiratory unit for investigation of the recurrent or severe nature of the ALTE. The bronchoscopies were therefore performed in the index and control groups to define anatomical and physiological normality or abnormality of the nasal space, larynx, trachea, bronchi, and secretions. During these assessments bronchoalveolar lavage was performed and fluid sent for differential cytology, including lipid laden macrophages, as well as for surfactant assays. Where repeat bronchoscopic procedures were carried out, the procedure was not the primary reason for an anaesthetic. Here the bronchoscopy was performed during the same anaesthetic for procedures such as grommet placement and replacement, cystoscopy, and revision of tracheostomy stoma and the airway before decannulation, or because of associated tracheostomy problems such as granuloma.

The bronchoscopy was carried out by one of the authors who is an experienced paediatric bronchoscopist. A halothane gaseous anaesthetic was used, the vocal cords sprayed with xylocaine and a Pentax $3.5 \mathrm{~mm}$ flexible scope was passed and wedged into a lower lobe bronchus where a bronchial lavage was performed using $2 \mathrm{ml} / \mathrm{kg}$ warm normal saline instilled in two aliquots via the wedged bronchoscope in the lobar bronchus. Samples of the lavage fluid recovered by suction were immediately shaken with liquid chloroform to elutriate lipid components of surfactant and to prevent biochemical degradation. All procedures were carried out with the same instrument and by the same bronchoscopist. Each sample was assayed by scientists unaware of the diagnosis.

\section{CONSENT}

Informed parental consent was obtained for the diagnostic bronchoscopy and bronchoalveolar lavage, as well as for the use of these investigatory results in the scientific forum. Approval for this study was granted by the Mater Children's Hospital research and ethics committee.

\section{SURFACTANT ASSAYS: HYSTERESIS TESTS}

The lipid and other hydrophobic components of surfactant in the recovered sample were extracted from the lavage fluid with chloroform and stored on ice. The aqueous layer was later decanted and the chloroform evaporated to dryness and redissolved in hexane/ethanol for deposition of the surfactant as a monolayer on the saline pool of a Langmuir trough. The evaluation of physical properties was carried out by a standard procedure whereby the surface area (A) of the monolayer was cycled over a ratio of $5: 1$ while its surface tension $(\gamma)$

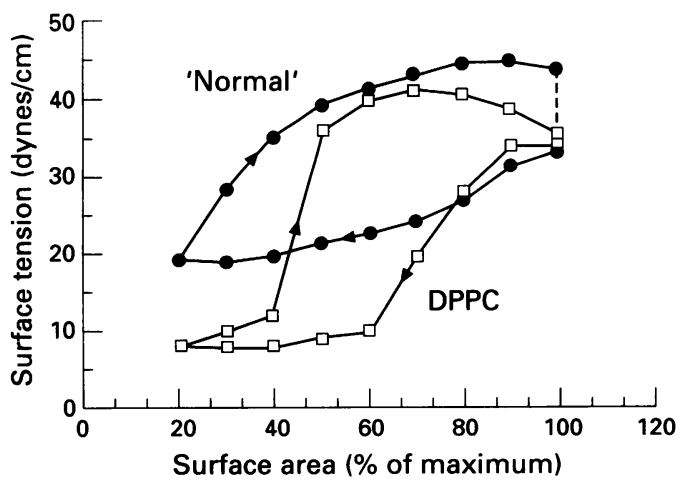

Figure 1 A typical plot of surface tension $(\gamma)$ versus area $(A)$ of the pool of a Langmuir trough for the first cycle of monolayers of pure DPPC and surfactant extracted from a lung lavage sample of a normal infant. Note the standard clockwise direction of rotation of the $\gamma: A$ loop characteristic of normal hysteresis. 


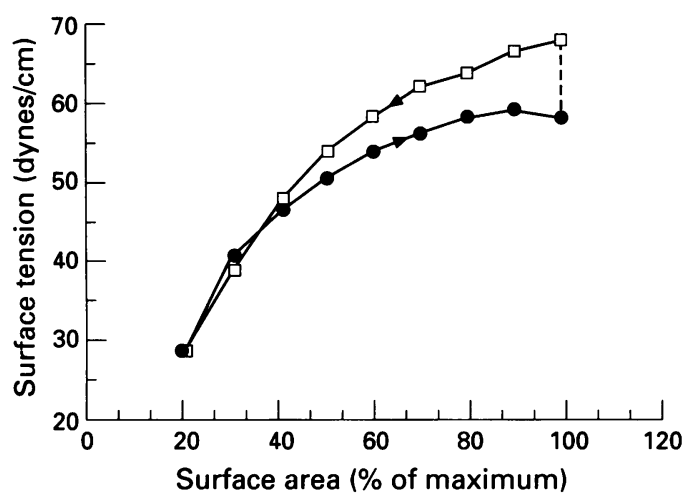

Figure 2 A plot of surface tension $(\gamma)$ versus area $(A)$ of the pool of a Langmuir trough for the first cycle of a monolayer of surfactant extracted from a lung lavage sample of an index case. Note that the $\gamma: A$ loop now cycles in an anticlockwise direction with hysteresis inverted (reversed) relative to normal (fig 1).

was measured by the Wilhelmy method using a platinum 'flag'. All studies were carried out at room temperature. Plots of $\gamma$ against $\mathrm{A}$ normally reveal a wide loop that cycles in the clockwise direction. Any loop that failed to do so either in toto (anticlockwise) or in part (figure of eight) was classified as abnormal. Representative loops are shown in figs $1-4$.

Total phospholipid is determined as the phosphorus content of the chloroform extract by use of perchlorate to oxidise all elemental phosphorus in the residue to phosphate, which is then quantified colourimetrically as phosphomolybdate employing a spectrophotometer.

\section{STATISTICS}

Descriptive statistics and the Fisher's exact test were used for analysis of the data. A p value of $\leqslant 0.05$ was regarded as significant.

\section{Results}

There were 23 index cases and 26 controls whose diagnoses or presenting features are shown in the table. The median age of onset of ALTE was 1 month (range 1-9 months). The

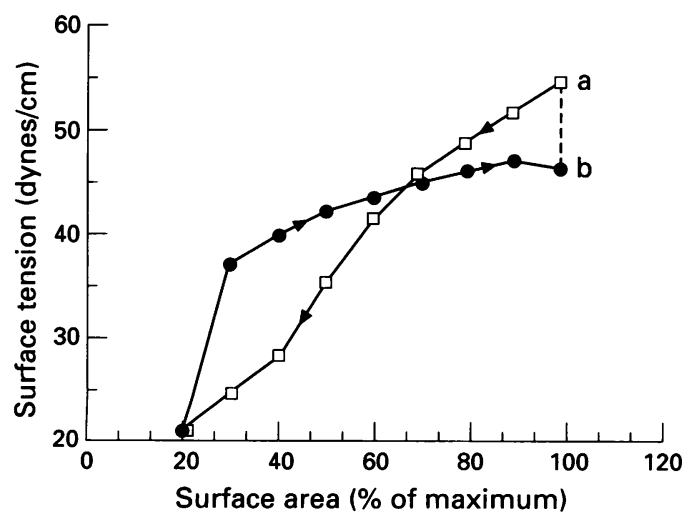

Figure $3 A$ plot of surface tension $(\gamma)$ versus area $(A)$ of the pool of a Langmuir trough for the first cycle of a monolayer of surfactant extracted from a lung lavage sample of an index case. Note how $\gamma: A$ hysteresis is partially inverted (reversed) by comparison with a normal clockwise loop (fig 1) to give a figure of eight. The degree of inversion is maximal at the initial (100\%) area as quantified by $(a-b)$.

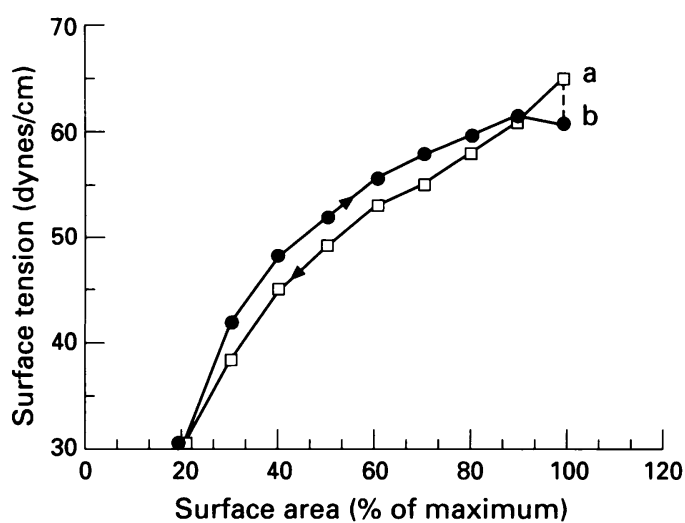

Figure $4 A$ repeat plot of surface tension $(\gamma)$ versus area $(A)$ of the pool of a Langmuir trough for the first cycle of a monolayer of surfactant extracted from a lung lavage sample of an index case. In the first test this in fact gave an inversion $(a-b)$ of only 0.8 dynes $/ \mathrm{cm}$ at $100 \%$ area and was one of two such cases classified as normal in the table. In the repeat test it can be seen how the inversion is clearly significant with $a=64 \cdot 7$ dynes $/ \mathrm{cm}$ and $b=60.6$ dynes $/ \mathrm{cm}$ when these would have been unequivocally classified as index cases.

median age (range) at which the bronchoscopy (test) was performed for index cases and controls was 8 months (range 1-120) versus 7.5 months (range 1-120) ( $>0.05$, NS). The reason for such a disparity in the time of the first ALTE and the time at which bronchoscopy was performed is due to the fact that a number of the infants and children were referred for assessments long after their first ALTE. The severity of the ALTE varied from mild without cyanosis $(n=2)$ to severe with metabolic acidosis $(n=6)$. Four of these infants survived and two died from SIDS. Fourteen index cases experienced recurrent bouts of cyanosis but were never documented as requiring hospital resuscitation for metabolic acidosis or respiratory failure. Four children had a clinical pattern consistent with prolonged expiratory apnoea. Four were classified as secondary ALTE and 12 as primary ALTE. Six controls also experienced apnoea and cyanosis as a result of severe airway obstruction.

\section{SURFACTANT ASSAYS}

The mean (SEM) amount of surfactant available for assay was $4.2(1.6) \mathrm{mg}$ in the index cases and $4.7(1.3) \mathrm{mg}$ for the controls. Four index cases and one control had surfactant levels $<0 \cdot 1 \mathrm{mg}$. These were regarded as 'low' concentrations of surfactant.

\section{SURFACE TENSION: SURFACE AREA LOOP} ASSESSMENTS

Of the 23 index cases, 17 had figure of eight type loop shapes, four anticlockwise loops, and two normal loops (figs 1-3). Loop shape classification clearly separated index cases from controls: there being a highly significant difference (Fisher's exact test $p \leqslant 0.0001$ ). On repeat testing, the normal loops were regarded as abnormal but were not included as abnormals in the statistical analysis. The three infants who had ALTE and were siblings of SIDS, all had abnormal loops. 
Age of onset of ALTE, age at bronchoscopy, and presenting features of index and control cases

\begin{tabular}{|c|c|c|c|}
\hline $\begin{array}{l}\text { Case } \\
\text { no }\end{array}$ & $\begin{array}{l}\text { Age at onset of } \\
\text { ALTEs (months) }\end{array}$ & $\begin{array}{l}\text { Age at bronchoscopy/ } \\
\text { lavage (months) }\end{array}$ & Presenting feature \\
\hline \multicolumn{4}{|c|}{ Index cases } \\
\hline 1 & 1 & 3 & Recurrent apnoea/cyanosis \\
\hline 2 & 1 & 4 & Recurrent choking/cyanosis \\
\hline 3 & 1 & 120 & Central alveolar hypoventilation. PEA \\
\hline 4 & 3 & 3 & Severe apnoea, acidosis \\
\hline 5 & 2 & 11 & Recurrent alarming. Apnoea of infancy \\
\hline 6 & 1 & 1 & Recurrent choking. No cyanosis \\
\hline 7 & 9 & 9 & Apnoea/no cyanosis \\
\hline 8 & 2 & 32 & Apnoea/cyanosis \\
\hline 9 & 9 & 9 & Tracheomalacia. Apnoea \\
\hline 10 & 1 & 1 & Severe apnoea, acidosis \\
\hline 11 & 2 & 5 & Apnoea/cyanosis. Mechanical ventilation \\
\hline 12 & 1 & 9 & Recurrent apnoea/cyanosis \\
\hline 13 & i & 8 & Recurrent apnoea/cyanosis. Reflux \\
\hline 14 & 3 & 4 & Apnoea/cyanosis, acidosis \\
\hline 15 & 4 & 20 & Apnoea/cyanosis \\
\hline 16 & 1 & 76 & Recurrent apnoea/cyanosis. PEA \\
\hline 17 & 1 & 30 & Recurrent apnoea/cyanosis \\
\hline 18 & 1 & 8 & Apnoea/cyanosis. Reflux \\
\hline 19 & 1 & 1 & Recurrent apnoea/cyanosis \\
\hline 20 & 1 & 6 & Recurrent apnoea/cyanosis. Acidosis. PEA \\
\hline 21 & 1 & 3 & Recurrent apnoea/cyanosis \\
\hline 22 & 8 & 8 & SIDS \\
\hline 23 & 1 & 1 & SIDS \\
\hline \multicolumn{4}{|c|}{ Control cases } \\
\hline 1 & & 5 & Pulmonary hypoplasia. Tracheostomy \\
\hline 2 & & 2 & Haemangioma. Tracheostomy \\
\hline 3 & & 7 & Subglottic stenosis. Cerebral palsy \\
\hline 4 & & 22 & Subglottic stenosis \\
\hline 5 & & 22 & Laryngotracheo bronchitis. Cricoid split \\
\hline 6 & & 5 & Tracheomalacia. Bronchitis \\
\hline 7 & & 9 & Dynamic tracheomalacia \\
\hline 8 & & 120 & Scoliosis - ventilated \\
\hline 9 & & 3 & Laryngomalacia \\
\hline 10 & & 6 & Intractable seizure - ventilated \\
\hline 11 & & 9 & Laryngomalacia. Tracheomalacia \\
\hline 12 & & 8 & Tracheomalacia. Vascular ring \\
\hline 13 & & 23 & Tracheomalacia \\
\hline 14 & & 8 & Tracheomalacia. Laryngomalacia \\
\hline 15 & & 3 & Laryngomalacia \\
\hline 16 & & 28 & Laryngomalacia. Bronchitis \\
\hline 17 & & 7 & Laryngomalacia. Tracheostomy \\
\hline 18 & & 1 & Laryngomalacia. Tracheomalacia \\
\hline 19 & & 9 & Laryngomalacia. Reflux \\
\hline 20 & & 2 & Laryngomalacia. Bronchitis \\
\hline 21 & & 2 & Laryngomalacia \\
\hline 22 & & 76 & Viral encephalopathy - ventilated \\
\hline 23 & & 2 & Laryngomalacia \\
\hline 24 & & $7 \overline{2}$ & Laryngomalacia. Bronchomalacia \\
\hline 25 & & 28 & Post foreign body atelectasis \\
\hline 26 & & 7 & Laryngomalacia \\
\hline
\end{tabular}

REPEAT LOOP ASSESSMENTS: REPRODUCIBILITY OF FINDINGS

In order to gain some understanding of the reproducibility of the initial findings, repeated testing of six index cases over a four to 12 month period was possible as the infants were undergoing elective surgery requiring a general anaesthetic. There was $100 \%$ concordance; that is, the loop abnormality was sustained over time.

\section{SLEEP STUDIES}

Six infants had abnormal sleep studies in that there were significantly more desaturations and lower minimum saturations recorded in rapid eye movement sleep than normal infants of the same age, as recorded in a previous study in our laboratory. ${ }^{13}$ The desaturations in rapid eye movement sleep were attributed to combinations of dysrhythmic respirations, apnoeas, paradoxical respirations, and hypopnoeas.

\section{OUTCOME}

None of the infants with recurrent ALTE have died despite the abnormalities in surfactant. One infant with PEA has developed cerebral palsy as a result of recurrent and severe hypoxic ischaemic events. Most with recurrent ALTE and PEA have continued to have some form of ALTE beyond the time of assessment and into the second year of life. Four of six children appeared to be improved with oxygen treatment, however, there was not complete resolution of ALTEs.

\section{Discussion}

This is the first study to show a definable abnormality in the physical properties of surfactant in children and infants with ALTE, PEA, and SIDS. While other studies have reported abnormalities in the chemical composition of surfactant, they did not report in detail the physical properties of the surfactant despite the reporting of variations in the chemical composition of their samples. ${ }^{3-5} 78$ Indeed it is feasible that they were overlooked or regarded as artefact as the degree of hysteresis does change after the first loop even in the normal situation. ${ }^{14}$ In our patients the reason for the abberation in loop shape and cycle direction change is unclear. It may of course be due to an aberration in the multiplicity of factors that govern adsorptive properties of surfactant in contact with the platinum flag as manifest by a change in contact angle, ${ }^{12}$ but it is unlikely to be related to the quantity of surfactant as our results were independent of surfactant concentration. Whether these findings are representative of the in vivo situation cannot be answered but it would seem most likely that such changes in physical properties are real, particularly as they were totally reproducible over long periods of time. As surfactant is the major 'governor' of surface and recoil forces within the lung at all but high volumes, it then would seem reasonable to assume that it might have a role in 'receptor triggering' and generally influencing afferent neural feedback to the brainstem. Given the nature of the loop abnormalities, it would then be possible for the receptors to 'read' the surface tension as being higher than is normal at low lung volumes and thus risk the brain's interpretation that the lung is in an inspiratory mode when, in reality, it is in an expiratory mode. As respiratory events are most common in rapid eye movement sleep, ${ }^{15}$ the controller system is even more likely to be vulnerable as the patient lung volumes, oxygen saturation state, and hypoxic and hypercapnoeic thresholds fluctuate as do the frequency of apnoeas and dysrhythmic respirations that characterise this sleep state. It is of some importance that six of 12 infants who had polysomnographic studies recorded significantly more desaturations and a greater degree of desaturation than recorded in normal infants of comparable age studied within our laboratory. These findings would be consistent with a disorder of functional residual capacity regulation.

Indeed with these arguments, it is then not necessary to postulate atelectasis in vivo or even abnormalities in lung compliance ${ }^{16}$ as one would expect the reverse in this situation as the actual surface tension could be maintained 
at values above that expected for the normal expiratory cycle. This would provide the potential for the recurrent apnoea that characterises these children and the rapid eye movement sleep state dominance of apnoea without necessarily having sustained abnormalities in compliance as implied by Fagan and Milner. ${ }^{16}$ The sustainability of our laboratory findings over time supports these contentions as do the clinical findings of recurrent apnoea extending into the childhood years in some of the individuals as shown by the age distribution in the table. Also it is unlikely that these pathophysiological effects could be sustained in the presence of more mature controller systems and more mature respiratory mechanics where it could be expected that the influence of chest wall deformity during the respiratory cycle would be lost and expiratory reserve volumes are increased thus negating the effects of a small change in compliance. While there are no current data to support a potential role of surfactant in neural receptor function, there is information supporting this contention in the organs of other species. ${ }^{17}$

While only four infants had non-idiopathic ALTE, most reports on ALTE reveal a significant number in whom other potential causes can be found. ${ }^{18}$ This being so, our findings would allow for a more unifying hypothesis and a way of differentiating those at 'true risk'.

\section{Conclusion}

This study has shown consistent abnormalities in the physical properties of surfactant in children with recurrent ALTEs and, while it is unclear at this stage as to what factors are producing these abnormalities, these findings may prove to be of great clinical importance in defining as well as differentiating ALTEs cases from other disorders that share similar presentations. Also these findings could prove to be an important way of identifying children at risk of ALTEs or even SIDS, and combined with previous publications ${ }^{3-10}$ support the possibility of a disease continuum or a common unifying factor for ALTEs and SIDS.

We thank the University of New England department of physiology and Ms Abigail Morris, the scientist who performed the surfactant assays.

1 Hoffman HJ, Damus K, Hillman L, Krongrad E. Risk factors for SIDS. Results of the National Institutes of Health and Human Development SIDS cooperative epidemiological study. Ann NY Acad Sci 1988; 533: 13-30.

2 Beckwith JB. Intrathoracic petechial hemorrhages: a clue to the mechanism of death in SIDS? Ann NYAcad Sci 1988; 533: 37-47.

3 Morley CJ, Hill CM, Brown BD, Barson AJ, Davis JA. Surfactant abnormalities in babies dying from sudden infant death syndrome. Lancet 1982; i: 1320-3.

4 Hill CM, Brown BD, Morley CJ, Davis JA, Barson AJ. Pulmonary surfactant. II. In sudden infant death synPulmonary surfactant. II. In sudden inf
drome. Early Hum Dev 1988; 16: 153-62.

5 Southall DP, Johnson P, Salmons S, et al. Prolonged expiratory apnoea: a disorder resulting in episodes of severe arterial hypoxaemia in infants and young children. Lancet 1985; ii: 571-7.

6 Southall DP, Samuels MP, Talbert DG. Recurrent cyanotic episodes with severe arterial hypoxaemia and intrapulmonary shunting: a mechanism for sudden death. Arch Dis Child 1990; 65: 953-61.

7 James D, Berry PJ, Fleming P, Hathaway M. Surfactant abnormality and the sudden infant death syndrome - a primary or secondary phenomenon? Arch Dis Child 1990; primary or $744-8$.

8 Gibson RA, McMurchie EJ. Changes in lung surfactant lipids associated with the sudden infant death syndrome. Australian Paediatric fournal 1985; (suppl): 77-80.

9 Gibson RA, McMurchie EJ. Decreased lung surfactant disaturated phosphatidylcholine in sudden infant death syndrome. Early Hum Dev 1988; 17: 45-55.

10 Hills B, Masters IB, O'Duffy J. Abnormalities of surfactan in children with recurrent cyanotic episodes. Lancet 1992 339: 1323-4.

11 Hills BA. A thermal surface phenomenon in the rabbit lung: possible basis for the conversion of heat into work. p Physiol (Lond) 1988: 402: 463-71.

12 Hills BA. Contact-angle hysteresis induced by pulmonary surfactants. $f$ Appl Physiol 1983; 54: 420-6.

13 Masters IB, Goes AM, Healy L, O'Neil M, Stephens D, Harris MA. Age related changes in oxygen saturation over the first year of life: a longitudinal study. $\mathcal{F}$ Paediatr Child Health 1994; 30: 423-8.

14 Barrow RE, Hills BA. Surface tension induced by dipalmitoly lecthin in vitro under physiological conditions. f Physiol (Lond) 1979; 297: 217-27.

15 Thach T. Apnoea and the sudden infant death syndrome. In: Saunders NA, Sullivan CE, eds. Lung biology in health and disease, sleep and breathing. New York: Marcel Dekker, 1994: 649-72.

16 Fagan DG, Milner AD. Pressure volume characteristics of the lungs in sudden infant death syndrome. Arch Dis Child 1985; 60: 471-85.

17 Sbarbati A, Cesesi E, Accordini C. Surfactant-like material on the chemoreceptorial surface of the frog's taste organ: an ultra structural and electon spectroscopic imaging study. F Struct Biol 1991; 107: 128-35.

18 Kahn A, Rebuffat E, Franco P, et al. Apparent life threatening events and apnoea of infancy. In: Backerman RC, Brouillette R, Hunt CE, eds. Respiratory control disorders in infants and children. Baltimore: Wilkins and Wilkins, 1992: 178-89. 